\title{
TüKaPo at SemEval-2020 Task 6: Def(n)tly not BERT: Definition Extraction using pre-BERT Methods in a post-BERT World
}

\author{
Madeeswaran Kannan* Haemanth Shanthi Ponnusamy ${ }^{\dagger}$ \\ Department of Linguistics \\ University of Tübingen, Germany
}

\begin{abstract}
We describe our system (TüKaPo) submitted for Task 6: DeftEval, at SemEval 2020. We developed a hybrid approach that combined existing CNN and RNN methods and investigated the impact of purely-syntactic and semantic features on the task of definition extraction, i.e, sentence classification. Our final model achieved a F1-score of 0.6851 in the first subtask.
\end{abstract}

\section{Introduction}

By all accounts, the first reliable English dictionary was written by Samuel Johnson ${ }^{1}$ and published on 15 April, 1755. It was 18 inches tall, 20 inches wide when opened, and contained 42,773 entries. It took Dr. Johnson 7 years to complete, and yet it was missing the word contrafibularity, amongst others. ${ }^{2}$ In the 265 years that have passed since, the world has evolved at a blisteringly fast pace with technology integrating itself ever more closely with our lives. However, the compilation and maintenance of dictionaries and lexicons - one of the most important and authoritative sources of meaning - continues to be the exclusive field of domain experts and lexicographers. Nevertheless, with the recent advances in natural language processing, this area - like many others that deal with human language - has seen a growing interest in automating the development of such resources.

Definition extraction is defined as the automatic identification of definitional knowledge in text, modeled as a binary classification problem between definitional and non-definitional text. In the early days of definition extraction, rule-based approaches leveraging linguistic features showed promise. Westerhout (2009) used a combination of linguistic information (n-grams, syntactic features) and structural information (position in sentence, layout) to extract definitions from Dutch texts. Such approaches, however, were found to be dependent on language and domain, and scaled poorly. Later research incorporated machine learning methods to encode lexical and syntactic features as word vectors (Del Gaudio et al., 2014). Noraset et al. (2017) tackled the problem as a language modelling task over learned definition embeddings. Espinosa-Anke et al. (2015) derive feature vectors from entity-linking sources and sense-disambiguated word embeddings. More recently, Anke and Schockaert (2018) use convolutional and recurrent neural networks over syntactic dependencies to achieve very good results on the WCL and W00 datasets (Navigli and Velardi, 2010; Jin et al., 2013).

This paper describes our approach of combining existing methods over state-of-the-art techniques that involve the use of contextualized word embeddings such as ELMo (Peters et al., 2018) and BERT (Devlin et al., 2018) in an attempt to determine if the former still offer avenues of optimization that can help them perform competitively with the latter.

\footnotetext{
*mkannan@sfs.uni-tuebingen.de

$\dagger$ haemanth.santhi-ponnusamy@student.uni-tuebingen.de

This work is licensed under a Creative Commons Attribution 4.0 International License. License details: http:// creativecommons.org/licenses/by/4.0/.

${ }^{1}$ https://en.wikipedia.org/wiki/A_Dictionary_of_the_English_Language

${ }^{2}$ Aardvark was another.
} 


\subsection{Task Background}

The DeftEval shared task (Spala et al., 2020) is based around the English-language DEFT (Definition Extraction From Texts) corpus (Spala et al., 2019). It consists of annotated text extracted from the following semi-structured and free-text sources. Compared to similar existing definition extraction corpora such as WCL (Navigli and Velardi, 2010) and W00 (Jin et al., 2013), the data offered by the DEFT corpus is larger in size (23,746 sentences; 11,004 positive annotations) while also providing finer-grained feature annotations.

The shared tasks consists of three subtasks: 1) Sentence Classification (classify if a sentence contains a definition or not), 2) Sequence Labeling (label each token with BIO tags according to the corpus specification), and 3) Relation Classification (label the relations between each tag according to the corpus specification). We participated in the first subtask. The test data for the first subtask is presented in the following format:

\section{[SENTENCE] [BIN_TAG]}

BIn_tAg is 1 if SENTENCE contains a definition, 0 otherwise. During training for the first subtask, the training and development datasets were converted into the same format as the test dataset using a script provided with the corpus. A positive label was associated with every sentence that contained tokens with B-Definition or I-Definition tags; all other sentences were associated with a negative label.

\section{System Overview}

\subsection{Baseline}

We developed and iterated on both LSTM-based (Hochreiter and Schmidhuber, 1997) recurrent and convolutional (O'Shea and Nash, 2015) neural network models. Our RNN architecture is a network of a single bidirectional LSTM layer followed by two feed-forward layers and a final sigmoid-activated read-out layer. This architecture is implemented by model $B L-R N N$ (Baseline RNN) whose input layer accepts sequences of feature vectors. Our hybrid-CNN architecture is implemented by model $B L-C N N$ (Baseline CNN), which is based on the work by Anke and Schockaert (2018). It accepts feature vector sequences that are passed through a one-dimensional convolutional filter and a max-pooling layer, followed by a single BiLSTM and read-out layers. The intuition behind combining convolutional and recurrent layers is to leverage the implicit local feature-extraction performed by the convolutional layers to refine the final representation passed to the recurrent layer, which accounts for global features. The input sequences are composed as concatenations of vectors of individual features at the token level, resulting in a homogenous representation, e.g. each token is encoded as the concatenation of a $n$-dimensional word vector, a $m$-dimensional one-hot encoded POS tag vector, etc.

We conducted several experiments with the above two architectures and iterated on successful models. The provided corpus was pre-split into train and dev splits. A 90-10 split was performed on the train split to generate the validation set; the $\operatorname{dev}$ split was used as the test data as-is. All models were trained for 100 epochs with an early-stopping mechanism that monitored the validation loss over the last 10 epochs. Batch size was set to 128 , and ADAM (Kingma and $\mathrm{Ba}$, 2014) was used as the binary cross-entropy optimizer. URLs were stripped from token sequences as a preprocessing step. Sentences were parsed with $\mathrm{spaCy}^{3}$ using the en_core_web_lg model ${ }^{4}$ to obtain POS tag sequences and dependency relation data.

The results of our experiments are listed in table 1 . The reported figures were averaged over three iterations of each experiment.

\footnotetext{
${ }^{3}$ https://spacy.io/

${ }^{4}$ https://spacy.io/models/en\#en_core_web_lg
} 


\begin{tabular}{|c|c|c|c|c|c|c|}
\hline Experiment & Model & Word Embeddings & Features & Precision & Recall & F1-Score \\
\hline $\begin{array}{l}\text { 1. W/o Semantic } \\
\text { Information }\end{array}$ & BL-RNN & - & POS + Deps & 0.56 & 0.75 & 0.64 \\
\hline \multirow{9}{*}{$\begin{array}{l}\text { 2. W/ Semantic } \\
\text { Information }\end{array}$} & \multirow{3}{*}{ BL-RNN } & Self-trained & \multirow{5}{*}{ Tokens } & 0.74 & 0.60 & 0.66 \\
\hline & & Glove & & 0.70 & 0.64 & 0.67 \\
\hline & & $\mathrm{w} 2 \mathrm{v}$ & & 0.70 & 0.61 & 0.65 \\
\hline & \multirow{2}{*}{ BL-CNN } & Glove & & 0.78 & 0.59 & 0.67 \\
\hline & & $\mathrm{w} 2 \mathrm{v}$ & & 0.77 & 0.51 & 0.61 \\
\hline & \multirow{2}{*}{ BL-RNN } & Glove & \multirow{4}{*}{ Tokens + Deps } & 0.74 & 0.63 & 0.68 \\
\hline & & $\mathrm{w} 2 \mathrm{v}$ & & 0.71 & 0.60 & 0.65 \\
\hline & \multirow{2}{*}{ BL-CNN } & Glove & & 0.72 & 0.62 & 0.67 \\
\hline & & $\mathrm{w} 2 \mathrm{v}$ & & 0.72 & 0.58 & 0.64 \\
\hline \multirow{8}{*}{$\begin{array}{l}\text { 3. Effect of punctuation \& } \\
\text { dependency relations }\end{array}$} & \multirow{5}{*}{ BL-RNN } & \multirow{8}{*}{ Glove } & Tokens + POS & 0.75 & 0.62 & 0.68 \\
\hline & & & Tokens + Deps + POS & 0.76 & 0.58 & 0.66 \\
\hline & & & Tokens + POS + Punct & 0.76 & 0.64 & 0.69 \\
\hline & & & Tokens + Deps + POS + Punct & 0.76 & 0.62 & 0.68 \\
\hline & & & Tokens + POS & 0.74 & 0.65 & 0.69 \\
\hline & \multirow{3}{*}{ BL-CNN } & & Tokens + Deps + POS & 0.77 & 0.67 & 0.71 \\
\hline & & & Tokens + POS + Punct & 0.77 & 0.64 & 0.70 \\
\hline & & & Tokens + Deps + POS + Punct & 0.79 & 0.62 & 0.70 \\
\hline \multirow{2}{*}{ 4. Final Model } & \multirow{2}{*}{ FINAL-HYBRID } & \multirow{2}{*}{ Glove } & Tokens + POS + Punct & 0.77 & 0.64 & 0.70 \\
\hline & & & Tokens + Deps + POS + Punct & 0.75 & 0.70 & 0.73 \\
\hline
\end{tabular}

Table 1: Results of experiments performed on the baseline \& final models

\subsection{Influence of Syntactic \& Semantic Information}

Our initial experiments were premised on the hypothesis that neural definition extraction can be primarily modelled on morphosyntactic features while excluding or restricting the use of semantic and lexical information. By limiting the influence of semantics, we expected to train a model that generalized well over multiple domains by virtue of being less susceptible to lexical cues that could potentially act as distractors. To test this, we trained multiple models on a combination of (embedded) word token, POS and dependency relation features. With the exception of one model that trained its own word embeddings, word embedding matrices of other models with word token features were initialized with 300-dimensional pre-trained GloVe (Pennington et al., 2014) and word2vec (Mikolov et al., 2013) embeddings respectively. The GloVe and $\mathrm{w} 2 \mathrm{v}$ embeddings were trained on the Common Crawl and Google News corpora respectively.

Most interestingly, the model that was exclusively trained on syntactic information was the one that performed the worst. Virtually all other models out-performed the syntax-only model even when they were only trained on word tokens. This fundamentally proved our hypothesis to be flawed, further corroborated by the minimal effect of network architecture on the results. These findings indicated that syntactic features were the least informative when used by themselves and the most informative when used in concert with semantic information provided by word embeddings. The corollary of the same suggests that word embeddings - pre-trained or otherwise - are able to approximate rudimentary information about syntax that would would otherwise be offered by syntactic features like part-of-speech tags. It also follows that combining both kind of features in a complementary manner should enable the model to perform better.

\subsection{Feature Modelling}

Building upon the findings of the previous experiments, we tested the effect of combining punctuation and part-of-speech tags. It was immediately evident that replacing the PUNCT POS tag with the punctuation character occurring at that position had a positive effect on the model's performance. Beyond the implicit increase in information offered by the actual character, it also reaffirms the importance of syntactic 


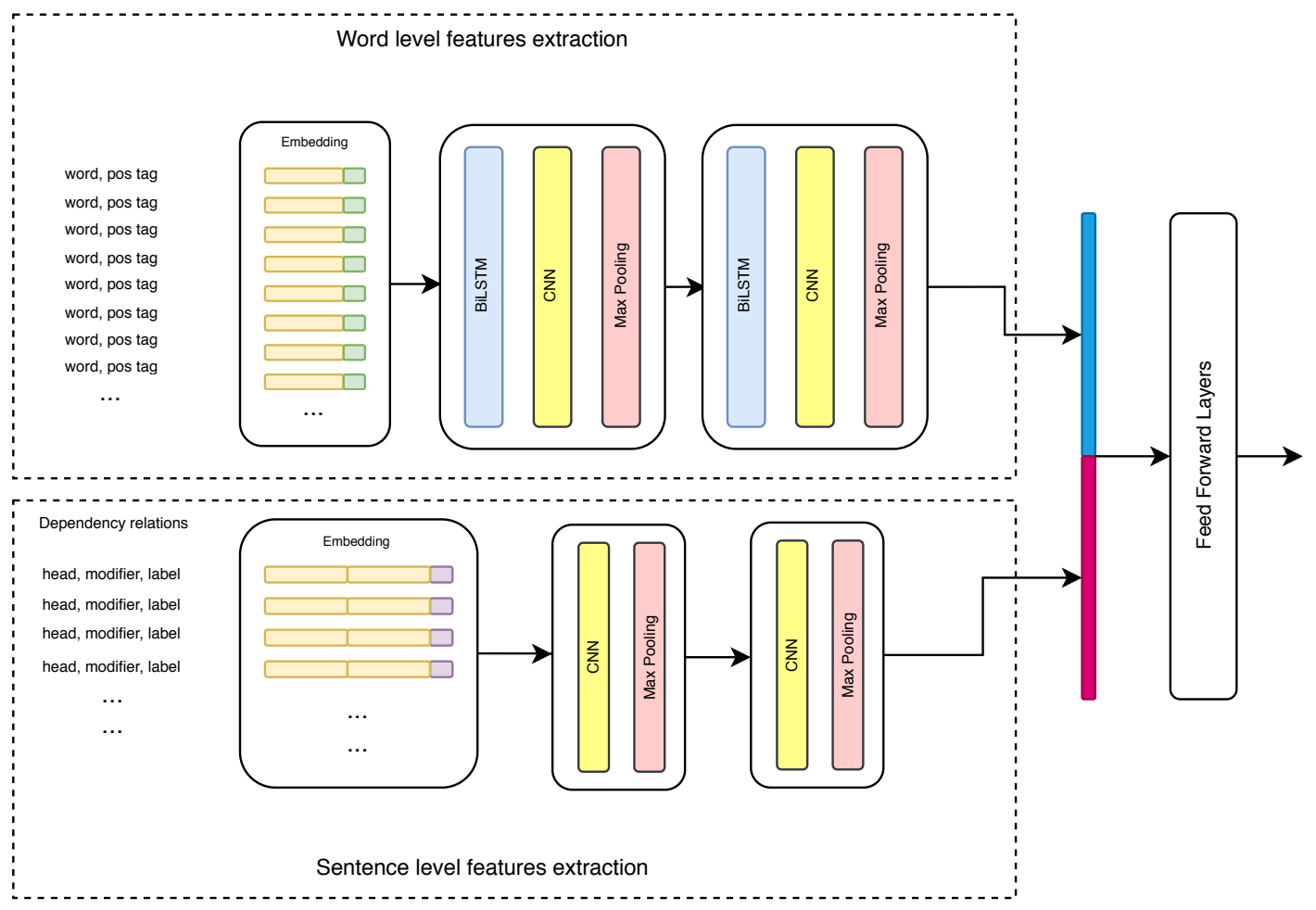

Figure 1: Final architecture

features in this task. The addition of dependency relation features, however, had a less-immediately obvious impact. The RNN model saw a reduction in performance while the hybrid-CNN model fared better. Upon further investigation, we determined that the input encoding scheme's attempt to homogenize feature vectors across disparate features, viz., combining sequential (token-level) features (token, POS) with non-sequential (sentence-level) features (dependencies), actually hindered the recurrent model from optimally exploiting the former. With this key insight, we were able to rearchitect our model to learn a representation that composes both token and sentence-level features in an separate but efficient manner.

\subsection{Final Architecture}

Our final architecture is informed by the findings of our previous experiments. It accepts three inputs: At the token-level, both token and part-of-speech tags (w/t punctuation) are used. Pre-trained GloVe embeddings are used for tokens, while embeddings for POS tags are learned on-the-fly. The concatenation of both embeddings is passed through two "feature extraction units" that consist of a BiLSTM (to target sequence/global information) and a 1D-Conv + MaxPool layer (to target local information). At sentence-level, dependency information is encoded as the concatenation of the embeddings of the head word, modifier word and dependency label of each relation. This is connected to two stripped-down "feature extraction units" without the BiLSTM layer, since dependency relations are sequentially independent. Finally, the extracted representations of both token and sentence-level features are concatenated and connected to a feed-forward layer and then a read-out layer.

The separation of feature-extraction at token and sentential levels allows their information to be combined at a higher level in the network. And we indeed see a marked improvement when this model is trained with dependency information. The model achieved a best F1-score of 0.76 during development.

\section{Results \& Discussion}

The final model achieved a positive-class F1-score of 0.6851 , ranking 47th out of 56 submissions for the first subtask. While the model under-performed in a substantial departure from our expectations, we identified factors that may have contributed to it. Since the gold-standard data for the test set was 


\begin{tabular}{|c|c|c|c|}
\hline \multirow{2}{*}{\multicolumn{2}{|c|}{ Hyperparameter }} & \multicolumn{2}{|c|}{ Value } \\
\hline & & Token-Level & Sentence-Level \\
\hline \multirow{3}{*}{$\begin{array}{l}\text { Embeddings } \\
\text { Dim }\end{array}$} & Word & \multicolumn{2}{|c|}{300} \\
\hline & POS & \multicolumn{2}{|c|}{32} \\
\hline & Dep. Label & \multicolumn{2}{|c|}{32} \\
\hline \multirow{4}{*}{$\begin{array}{l}\text { Feature Extractor } \\
\text { Units } \\
\text { (Unit 1, Unit 2) }\end{array}$} & LSTM Units & 128,64 & - \\
\hline & Conv. Filters & 128,64 & 64,32 \\
\hline & Conv. Kernel Size & \multicolumn{2}{|c|}{3,3} \\
\hline & MaxPooling Pool Size & \multicolumn{2}{|c|}{2,2} \\
\hline \multicolumn{2}{|c|}{ L2 Regularization $\beta$} & \multicolumn{2}{|c|}{0.001} \\
\hline \multicolumn{2}{|c|}{ Feed-forward Units } & \multicolumn{2}{|c|}{24} \\
\hline
\end{tabular}

Table 2: Hyperparameters for the final model

not available at the time of publication, we based our statements on our analysis of the training corpus and the prediction results of the test data. During training, we noticed that the number of unique terms in the preprocessed corpus out-stripped the number of training samples (over $21 \mathrm{~K}$ unique tokens in approx. $17 \mathrm{~K}$ sentences), over $75 \%$ of which occurred only once. This inevitably results in a large pool of out-of-vocabulary words. Pre-trained word embeddings trained on a relatively small corpus would be unable to model the vocabulary of the DEFT corpus completely, particularly as the latter mostly comprises of domain-specific text. This could potentially be mitigated by restricting the vocabulary based on term frequency count, but care must be taken not to restrict it too much as definitions, by definition, are dependent on uniquely identifiable terms.

We also found several incongruities in the corpus where contradictions in annotations led to an ambiguous ground-truth. Consider the following sentences from the training corpus: "Organisms are individual living entities." and "Organelles are small structures that exist within cells." The first sentence was annotated with the positive class (contains a definition) even though the latter was not. Another similar albeit more ambiguous example: "Recall from The Macroeconomic Perspective that if exports exceed imports, the economy is said to have a trade surplus." and "If imports exceed exports, the economy is said to have a trade deficit." Here, the second sentence is tagged as containing a definition even though the first is not. While some of these ambiguities can be attributed to how the training data for the binary classification task is generated from the larger sequence-annotated corpus, there are many other counter-examples where the rationale behind the annotation is unclear. Such incongruities ultimately make it more challenging for the model to attain a clear and optimal generalization.

\section{Conclusion}

We presented our system for definition extraction whose pre-BERT methods achieved an admittedly pre-historic F1-score of 0.6851 in Task 6: DeftEval, subtask 1. Future work could potentially include the customization of the architecture to incorporate ensemble training, exploring the usage of more task-specific cues such as topical information, and perhaps even becoming one with the BERT side and using contextualized word embeddings - In light of our experiments with the combination of syntactic and semantic features, the ability of the model to implicitly reproduce the classical NLP pipeline (Tenney et al., 2019) makes it a natural fit for the task. However, not all languages and domains have the ample amount of text resources required to (pre-)train large Transformer-based models such as BERT, not to mention the increasing computational costs of training such models. Therefore, one should not lightly dismiss the advantages of linguistically-motivated, task-specific approaches in favour of more general, task-agnostic ones. 


\section{References}

Luis Espinosa Anke and Steven Schockaert. 2018. Syntactically aware neural architectures for definition extraction. In Proceedings of the 2018 Conference of the North American Chapter of the Association for Computational Linguistics: Human Language Technologies, Volume 2 (Short Papers), pages 378-385.

Rosa Del Gaudio, Gustavo Batista, and António Branco. 2014. Coping with highly imbalanced datasets: A case study with definition extraction in a multilingual setting. Natural Language Engineering, 20(3):327-359.

Jacob Devlin, Ming-Wei Chang, Kenton Lee, and Kristina Toutanova. 2018. Bert: Pre-training of deep bidirectional transformers for language understanding. arXiv preprint arXiv:1810.04805.

Luis Espinosa-Anke, Horacio Saggion, and Claudio Delli Bovi. 2015. Definition extraction using sense-based embeddings. In Gupta P, Banchs RE, Rosso P, editors. International Workshop on Embeddings and Semantics (IWES'15); 2015 Sept 15; Alicante, Spain.[Place unknown]:[CEUR]; 2015.[6 p.]. CEUR.

Sepp Hochreiter and Jürgen Schmidhuber. 1997. Long short-term memory. Neural computation, 9(8):1735-1780.

Yiping Jin, Min-Yen Kan, Jun Ping Ng, and Xiangnan He. 2013. Mining scientific terms and their definitions: A study of the ACL anthology. In Proceedings of the 2013 Conference on Empirical Methods in Natural Language Processing, pages 780-790.

Diederik P Kingma and Jimmy Ba. 2014. Adam: A method for stochastic optimization. arXiv preprint arXiv:1412.6980.

Tomas Mikolov, Kai Chen, Greg Corrado, and Jeffrey Dean. 2013. Efficient estimation of word representations in vector space. arXiv preprint arXiv:1301.3781.

Roberto Navigli and Paola Velardi. 2010. Learning word-class lattices for definition and hypernym extraction. In Proceedings of the 48th annual meeting of the association for computational linguistics, pages 1318-1327. Association for Computational Linguistics.

Thanapon Noraset, Chen Liang, Larry Birnbaum, and Doug Downey. 2017. Definition modeling: Learning to define word embeddings in natural language. In Thirty-First AAAI Conference on Artificial Intelligence.

Keiron O'Shea and Ryan Nash. 2015. An introduction to convolutional neural networks. arXiv preprint arXiv:1511.08458.

Jeffrey Pennington, Richard Socher, and Christopher D Manning. 2014. Glove: Global vectors for word representation. In Proceedings of the 2014 conference on empirical methods in natural language processing (EMNLP), pages $1532-1543$.

Matthew E Peters, Mark Neumann, Mohit Iyyer, Matt Gardner, Christopher Clark, Kenton Lee, and Luke Zettlemoyer. 2018. Deep contextualized word representations. arXiv preprint arXiv:1802.05365.

Sasha Spala, Nicholas A. Miller, Yiming Yang, Franck Dernoncourt, and Carl Dockhorn. 2019. DEFT: A corpus for definition extraction in free- and semi-structured text. In Proceedings of the 13th Linguistic Annotation Workshop, pages 124-131, Florence, Italy, August. Association for Computational Linguistics.

Sasha Spala, Nicholas Miller, Franck Dernoncourt, and Carl Dockhorn. 2020. Semeval-2020 task 6: Definition extraction from free text with the deft corpus. In Proceedings of the 14th International Workshop on Semantic Evaluation.

Ian Tenney, Dipanjan Das, and Ellie Pavlick. 2019. Bert rediscovers the classical nlp pipeline. arXiv preprint arXiv:1905.05950.

Eline Westerhout. 2009. Definition extraction using linguistic and structural features. In Proceedings of the 1st Workshop on Definition Extraction, pages 61-67. Association for Computational Linguistics. 